\title{
Methodische Untersuchungen zur quantitativen Mikrobestimmung von ATP in biologischem Material mit dem Firefly-Enzymsystem
}

\author{
Von D. A. Kalbhen und H. J. Кoch \\ Ans dem PharmakologischenInstitut der Rheinischen Friedrich-Wilhelms-Universilät Bonn (Direktor: Prof. Dr. R. Domenjoz)
}

(Eingegangen am 18. Februar 1967)

Die beschriebenen methodischen Untersuchungen umfassen die Extraktion von ATP aus Geweben und seine quantitative Bestimmung mit der Iuciferin-Lucifcrase-Reaktion im Flüssigkeits-Szintillationszähler. Dabci werden die Stabilität von ATP in I_ösungen und im Gewebe fost mortem sowic eine optimale Extraktionsmethode dargestellt. Die Bedingungen und Gesetzmäßigkeiten der Lumineszenzreaktion, ihre Spezifität und ihre mögliche Beeinflusssung durch Störfaktoren werden aufgeführt. Die große Anwendungsbreite der Extraktions- und Bestimmungsmethode, ihre Empfindlichkeit und leichte Durchführbarkeit ermöglichen ATP-Messungen mit hoher Genauigkcit und guter Reproduzierbarkeit.

The extraction of ATP from tissues and its quantitative measurement with the luciferin-luciferase reaction in a liquid scintillation counter was studied. The stability of ATP in solution and in post mortem tissues was investigated, and an optimal extraction is described. The conditions for the luminescent reaction, the laws that govern it, its specificity and the possibility of interference are discussed. Owing to the wide applicability of the extraction and determination method, its sensitivity and simplicity of operation, ATP can be measured with high accuracy and good reproducibility.

Im Rahmen der Untersuchung des Energiehaushaltes im Verlauf experimenteller Entzündungen interessierten wir uns für Veränderungen des Adenosintriphosphat(ATP)-Gehaltes im entzündeten Gewebe. Dabei war es notwendig, eine Extraktionsmethode und ein Verfahren zur analytischen Bestimmung von ATP auszuarbeiten, das auch bei Routinearbeiten exakte quantitative und gut reproduzierbare Werte lieferte. Zur qualitativen und quantitativen ATP-Bestimmung sind in der Literatur eine Vielzahl von Verfahren beschrieben (1). Neben chemischen Methoden, die die Schwerlöslichkeit bestimmter Phosphat- oder Adeninverbindungen ausnutzen, kennen wir ATP-Bestimmungen mit Hilfe von Ionenaustauschern $(2,3)$, wie sie auch in unserem Institut von Kalbhen (4) und Wirthaus (5) angewendet wurden. Im Bereich biochemischer Untersuchungen wurden Fermentreaktionen, an denen ATP essentiell beteiligt ist, zur Bestimmung dieser Verbindung herangezogen. Auf diesem Prinzip beruhen ATP-Bestimmungen z. B. mit Hexokinase ${ }^{1}$ ) (6) und Glycerat-Kinase. Andere Bestimmungen basieren auf dem enzymatischen Abbau von ATP z. B. durch Apyrase, Myosin-ATP-ase, Phosphatase oder Myokinase mit nachfolgender Analyse und Bestimmung der entstandenen Reaktionsprodukte. In jüngster Zeit hat besonders die Luciferase-Methode, die erstmals 1952 von Strehler und Totter (7) angewandt wurde, große Verbreitung gefunden. Über die Extraktion von ATP aus biologischem Material sind in der Literatur sehr viele - zum Teil sich widersprechende - Arbeiten erschienen. Während einige Autoren die Extraktion mit Trichloressigsäure oder Perchlorsäure empfehlen, wird von anderen vor der Säurehydrolyse gewarnt. Die im Gewebe vorhandenen ATP-asen können außer

1) Der Trivialname Hexokinase wird hier gebraucht für das Enzym ATP: D-hexose 6-phosphotransferase EC. 2.7.1.1; Glyceratkinase für ATP: D-Glycerate 3-phosphotransferaseEC. 2.7.1.3.1, Apyrase für ATP diphosphohydrolase EC. 3.6.1.5, Myosin-ATPase für ATP phosphohydrolase EC. 3.6.1.3, Phosphatase für Orthophosphoric monoester phosphohydrolase EC. 3.1.3.1 und 3.1.3.2, Myokinase für ATP: AMP phosphotransferase 2.7.4.3. durch Säure auch durch Hitze inaktiviert werden, worauf sich die „Heißwasser-Extraktion“ von ATP begründete. Aber auch bei diesen Verfahren kann es zur hydrolytischen Zersetzung von ATP kommen. Die unterschiedlichen ATP-Gehaltsangaben verschiedener Autoren dürften vor allem auf den unterschiedlichen Extraktionsverfahren beruhen. In den folgenden Ausführungen soll neben detaillierten Angaben über die quantitative Mikrobestimmung von ATP mit der Luciferase-Reaktion auch eine zuverlässige und leicht durchführbare Extraktionsmethode dargestellt werden, die in einer Vielzahl von Untersuchungen gut reproduzierbare Werte erbrachte.

\section{Methoden und Ergebnisse}

Material

Apparaturen: Kühlzentrifuge Typ „Universal-III-Ks“ der Fa. Christ, Osterode

Bühler-Homogenisator

Elektrisches Wasserbad

Flüssigkeits-Szintillations-ZählerModell 725 der Fa. Nuclear Chicago

Tiermaterial: Albino-Wistar-Ratten im Gewicht von $120-250 \mathrm{~g}$

Substanzen: Adenosintriphosphat als Natriumsalz Adenosindiphosphat als Natriumsalz

Creatinphosphat als Natriumsalz

d-ATP als Na-Salz

(alle Substanzen von $\mathrm{Fa}$. Boehringer, Mannheim) UTP von $\mathrm{Fa}$. Serva, Heidelberg

Glühwürmchenschwänze (Firefly Tails) gefriergetrocknet, ganz oder grob gepulvert von der $\mathrm{Fa}$. Serva, Heidelberg oder von der Worthington Chemical Corp., Frebold, N. J. USA.

Alle weiteren Substanzen und Reagenzien von der Fa. Merck, Darmstadt.

\section{Untersuchungen zur Stabilität von ATP-Lösungen}

Zur quantitativen Bestimmung von Adenosintriphosphat in biologischem Material ist es notwendig, daß das ATP sowohl in den Extrakten als auch in den Vergleichs- oder Standardlösungen stabil bleibt. In der Literatur ist aufgeführt (1), daß wäßr. Lösungen von ATP in hohen Konzentrationen stabil, in geringen dagegen instabil sind.

In unseren Untersuchungen wurden ATP-Lösungen von 80 $\mu \mathrm{g} / \mathrm{ml}$ in folgenden Medien auf ihre Beständigkeit geprüft: 
1. Aqua bidest. $\mathrm{pH} 6,5-7,0$

2. $0,1 \mathrm{~N}$ Phosphatpuffer $\mathrm{pH} 8,2$

3. $0,1 \mathrm{M}$ Glycinpuffer $\mathrm{pH} 9,8$

Die Lösungen wurden bei Zimmertemperatur über dic Dauer von $6 \mathrm{Stdn}$. aufbewahrt und die ATP-Konzentrationen nach verschiedenen Zeitabständen bestimmt.

Wie aus der Abbildung $1 \mathrm{zu}$ ersehen, ist die ATPKonzentration der wäßr. Lösung nach 3 Stdn. bereits um 50\% gesunken, während sịch das ATP im Phosphatpuffer $\mathrm{pH} 8,2$ wesentlich langsamer zersetzt und erst nach etwa 8 bis 9 Stdn. den $50 \%$-Wert erreichen wird. Dagegen konnten wir im Glycinpuffer $\mathrm{pH} 9,8$ über den Zeitraum von $6 \mathrm{Stdn}$. bei Zimmertemperatur keinen Abfall des ATP-Gehaltes feststellen. Auch in dem von uns später verwendeten Glycinpuffer pH11,0konnte während Aufbewahrungszeiten von mehreren Wochen keine Hydrolyse des ATP nachgewiesen werden, so daß wir unsere ATP-Standardlösungen stets mit diesem Puffer herstellten und aufbewahrten. Bei längerer Lagerung wurden die Standardlösungen eingefroren.

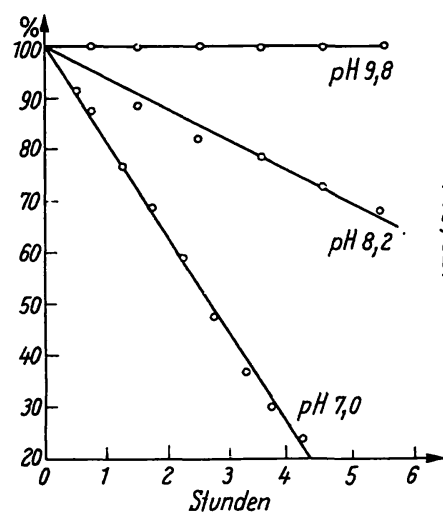

Abb. 1

Zeitlicher Zerfall von ATP in verschiedenen Lösungen

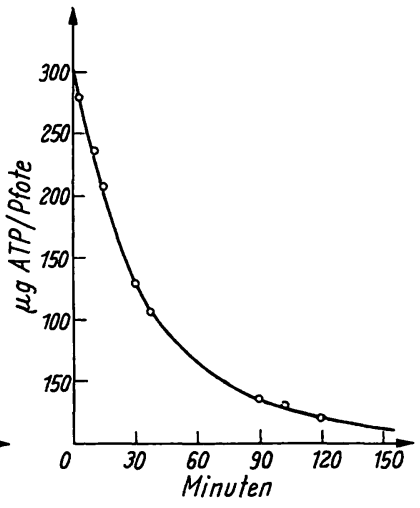

Abb. 2

Abnahme des ATP-Gehaltes in amputierten Rattenpfoten während der Lagerung bei Zimmertemperatur

\section{Extraktion von ATP aus biologischem Material}

Aufgrund unserer pharmakologischen Problemstellung wurden die vorliegenden methodischen Untersuchungen an amputierten Rattenpfoten durchgeführt. $\mathrm{Da}$ dieses Material aus heterogenen Geweben zusammengesetzt ist (bestehend aus Haut, Bindegewebe, Knochen, Sehnen, Knorpel), dürften die hier erarbeiteten Methoden auch leicht auf andere Gewebe, z. B. Leber, Gehirn, Herz usw., anwendbar sein.

Notwendige Voraussetzungen, um eine quantitative und reproduzierbare Extraktion aus biologischem Material zu erzielen, sind die optimale Zerkleinerung des Gewebes, die Blockierung des enzymatischen $\mathrm{Ab}$ baus des ATP durch ATP-asen und die Verhinderung hydrolytischer Prozesse im Extraktionsmedium.

Zur Extraktion von ATP ist Homogenisieren des Gewebes in kalter Perchlorsäure, kalter Trichloressigsäure und in heißem Wasser empfohlen worden. In allen Fällen kommt es dabei zu einer vollständigen Inaktivierung der gewebseigenen ATP-asen. Dagegen ist eine hydrolytische Spaltung des ATP besonders in den sauren Lösungen nicht ausgeschlossen. In unseren Versuchen wurde das Gewebe mit einem Bühler-Homogenisator zerkleinert. Von der
Methode, die Rattenpfoten unmittelbar post mortem in flüssigem Stickstoff einzufrieren und zu homogenisieten, nahmen wir bald Abstand, da sich die Pfoten nur in weitgehend aufgetautem $\mathrm{Zu}$ stand homogenisieren ließen, wobei stets ein unbefriedigender Zerkleinerungsgrad erreicht wurde. Nach dem Zentrifugieren des Homogenats und nach der Neutralisation des Extraktes mit kalter Kalilauge konnten wir nur noch Spuren von ATP in dem Pfotengewebe nachweisen. Auf die Extraktion mit kalter Trichloressigsäure mußten wir verzichten, da dieses Medium die von uns angewandte Bestimmungsmethode mit der Luciferin-Luciferase-Reaktion stört. Mit der Heißwassermethode konnten wir weitaus bessere Ergebnisse erzielen. In heißem Wasser ließ sich das Gewebe gut homogenisieren und ergab ATP-Werte von $\max .50 \mu / \mathrm{g}$ Pfote. Bedingt durch hydrolytische Prozesse in der wäßr. ATP-Extraktion konnte aber eine befriedigende Reproduzierbarkeit der Werte nicht erreicht werden.

Angeregt durch die oben aufgezeigte Stabilität der ATP-Lösungen in Glycinpuffer $\mathrm{pH} 11,0$ versuchten wir die Extraktion mit diesem Medium. Dabei gelang es, bei leichter und besserer Zerkleinerung des Gewebes stabile Extrakte zu gewinnen, deren ATP-Gehalt auch bei über 24-stdg. Lagerung nicht abnahm.

Unter Verwendung eines „Internal Standard“, wobei wir den Pfoten kurz vor der Homogenisation bekannte Mengen ATP zusetzten, konnten wir stets über $90 \%$ der zugefügten Menge wiederfinden, so daß ein nennenswerter enzymatischer oder hydrolytischer Abbau von ATP bei dieser Extraktionsmethode nicht auftrat. Der heiße alkalische Puffer ermöglicht zudem ein sehr günstiges Aufschließen des Gewebes.

Aufgrund unserer Untersuchungen konnten wir also folgendes Verfahren als optimal zur Extraktion von ATP aus biologischem Material ermitteln: Unmittelbar nach dem Tode werden 2-3 g Gewebe (in unserem Falle 2 Rattenpfoten) in $25 \mathrm{ml} 0,1 \mathrm{M}$ Glycinpuffer pH 11,0 , etwa $80-95^{\circ}$, für genạu 15 Sek. im BühlerHomogenisator bei etwa $50000 \mathrm{U} / \mathrm{Min}$. zerkleinert. Anschließend wird das Homogenat für 5 Min. auf dem siedenden Wasserbad erwärmt und danach durch ein Papierfilter filtriert. In dem weißlich trüben Filtrat ist das ATP lange haltbar und kann direkt oder nach Verdünnung mit Glycinpuffer bestimmt werden.

Bei Blut und Zellsuspensionen aus Zellkulturen ist eine Homogenisation nicht notwendig. Hierbei genügt ein inniges Mischen der Zellen mit dem heißen Puffer und anschließendes Erwärmen in dem Wasserbad, um eine optimale Extraktion von ATP zu erzielen.

\section{Untersucbungen ïber die Stabilität von $A T P$ im Gevvebe}

Da zwischen dem Töten der Versuchstiere und der Gewinnung und Wägung der zu untersuchenden Gewebe eine geringe Zeit verstreicht, erschien es uns notwendig, den Abfall dẹ ATP-Konzentration im Gewebe post mortem zu erfassen. Bekanntlich überlebt die einzelne Zelle mit ihrer eigenständigen Organisation und biochemischen Leistung den klinischen Tod des Individuums unter Umständen für eine längere Zeit. Die Ưberlebenșdauer hängt dabei sowohl von der Art der Zelle als auch von den funktionellen Eigenschaften des umgebenden Zellverbandes und des Mediums ab. Um den zeitlichen Verlauf des ATP-Zerfalls in den Rattenpfoten nach Amputation zu erfassen, wurden die amputierten rechten und linken Hinterpfoten von 8 männlichen Ratten nach verschieden langer Liegezeit (bei Zimmertemperatur) auf ihren ATP-Gehalt untersucht. Die Ergebnisse dieser Untersuchungen sind in Abbildung 2 dargestellt.

Daraus ișt $\mathrm{zu}$ entnehmen, daß es besonders in der ersten Zeit nach dem Tode (nach Unterbrechung der 
Sauerstoffversorgung des Gewebes und damit Stillstand der oxydativen Phosphorylierung) zu einer raschen Abnahme von ATP kommt. Nach 2 Stdn. war nut noch ein Zehntel der ursprünglichen ATPKonzentration nachweisbar. Diese Befunde machen deutlich, daß die Zeit zwischen Tötung der Tiere und Extraktion des ATP aus dem Gewebe möglichst kurz und in jedem Falle einheitlich sein muß. Verschieden lange Aufbereitungszeiten führen zwangsläufig zu unterschiedlichen Meßergebnissen.

Zur Methodik der ATP-Bestimmung mit dem Luciferin-Luciferase-Sy.stem der Glühwürmchen im Liquid-Scintillation Counter

Im Jahre 1887 entdeckte Durors (8) bei seinen Arbeiten über die lumineszierende Venusmuschel (Pholas dactylus) eine hitzestabile Substanz, die er "Luciferin" nannte, und ein hitzelabiles Enzym, das den Namen "Luciferase" erhielt. Es hat sich gezeigt, daß dieses "Luciferin-Luciferase-System" bei mehreren Tierarten und auch in einigen Pflanzen vorkommt und für die Lumineszenz mitverantwortlich ist. Allerdings haben Luciferin und Luciferase der verschiedenen Tierarten bzw. Pflanzen nicht dieselben chemischen Eigenschaften, wie z. B. McElroy (9) beim Vergleich der entsprechenden Substanzen aus Glühwürmchen (Photinus pyralis, im angelsächsischen Schrifttum „Firefly") und aus Cypridina bilgendorfii, einer Krebsart, nachwies. Außerdem werden zur Erzeugung des Lumineszenzphänomens neben Luciferin und Luciferase noch andere Substanzen benötigt, und diese wiederum variieren von Spezies zu Spezies.

Bedeutungsvoll war die Entdeckung McElroys, daß ein wäßriger Extrakt aus den Leuchtorganen von Glühwürmchen nach Abnahme der Lichtemission durch Zugabe von Adenosintriphosphat (ATP) wieder zum Leuchten gebracht werden konnte. McElroy und STREHLER (10) zeigten 1949, daß bei Glühwürmchenextrakten neben dem Enzym Luciferase, ATP, Sauerstoff und Wasser noch ein 2-wertiges Kation sowie Luciferin zur Lumineszenz nötig sind. Besonders geeignet erwies sich das Magnesiumion. Von GreEN und McElroy (11) wurde 1956 die Glühwürmchen-Luciferase und von Brtter und McElroy 1957 das GlühwürmchenLuciferin kristallin dargestellt (12). Als STREHLER und Totrer 1952 zum ersten Male diese Biolumineszenz zur Bestimmung von ATP in vitro anwendeten, erhielten sie bereits damals ausgezeichnete Ergebnisse (7).

Es ist charakteristisch für die Leuchtorgane der Glühwiirmchen, $\mathrm{da} B$ das aus ihnen gewonnene LuciferinLuciferase-System neben $\mathrm{H}_{2} \mathrm{O}, \mathrm{O}_{2}$ und $\mathrm{Mg}^{++}$zur Lichterzeugung $A T P$ benötigt. Die Luciferase wirkt lediglich als Katalysator bei der Oxydation reduzierten Luciferins zu Oxy-Luciferin, und letzteres ist dann verantwortlich für die Lumineszenz:

(1) ATP + Luciferin $\stackrel{\mathrm{Mg}^{++} \text {Lucilerase }}{\longrightarrow}$ Adenyl-Luciferin + Pyrophosphat

(2) Adenyl-Luciferin $\longrightarrow \mathrm{O}_{2} \longrightarrow$ Adenyl-Oxy-Luciferin $+\mathrm{H}_{2} \mathrm{O}$ + Licht
Strehler und McElroy (13) sowie andere Autoren haben sich bemüht, die sich aus dieser Reaktion ergebende Möglichkeit der ATP-Bestimmung mit Hilfe eines Extraktes aus den Leuchtorganen von Glühwürmchen durch Modifikation der Extraktionsverfahren optimal anzuwenden.

Extraktion des Luciferin-Luciferase-Systems aus den Leuchtorganen von Glühw ürmchen

Unseren eigenen Untersuchungen und den Angaben aus der Literatur entsprechend verwendeten wir zur Extraktion der Glühwürmchenschwänze einen $0,1 \mathrm{M}$ $\mathrm{Na}$-arsenat Puffer $\mathrm{pH} 7,4$, der aus $\mathrm{Na}_{2} \mathrm{HAsO}_{4} .7 \mathrm{H}_{2} \mathrm{O}$ und verd. Schwefelsäure hergestellt wurde. $50 \mathrm{mg}$ der im Kühlschrank aufbewahrten, gefriergetrockneten, ganzen oder grob gepulverten Firefly Tails wurden mit reinem Sand fein verrieben, zweimal mit je $10 \mathrm{ml}$ Puffer extrahiert und anschließend zentrifugiert. Der hellgelbe, klare Überstand wurde mit Puffer auf ein Endvolumen von $500 \mathrm{~m} l$ verdünnt. Vor Gebrauch wurde dem Enzymextrakt noch Magnesiumsulfat bis zur Endkonzentration von $0,02 \mathrm{M} \mathrm{MgSO}_{4}$ zugegeben. Andere $\mathrm{Mg}^{++}-\mathrm{Konzentrationen}$ erwiesen sich in unseren Versuchen als ungünstiger. Der so erhaltene Extrakt verlor bei Aufbewahrung im Kühlschrank schon nach wenigen Tagen an Aktivität und muß deshalb jeweils frisch angesetzt werden. $\mathrm{Da}$ die Enzymaktivität in den ersten Stunden besonders stark abfällt, empfiehlt es sich, den Extrakt vor der ersten Messung etwa 2 Stdn. bei Zimmertemperatur stehen zu lassen. Die einzuwiegende Menge von Glühwürmchenschwänzen kann variiert werden, da der Enzymgehalt der Leuchtorgane aus verschiedenen Lieferungen unterschiedlich sein kann. Außerdem richtet sie sich wie später noch näher erläutert wird - nach der ATPKonzentration des zu untersuchenden Objektes.

Messung der Biolumineszenz

Zur Messung der Lumineszenz bei der LuciferinLuciferase-Reaktion sind von früheren Untersuchern vor allem Photometer, Fluorimeter und später eigens zu diesem Zweck entwickelte Quantenzähler verwendet worden. Flüssigkeits-Stinzillations-Zähler, die zur Messung radioaktiver Substanzen (besonders für schwache Beta-Strahler) weite Verbreitung gefunden haben, besitzen neben einer ausgezeichneten elektronischen Meßsteuerung hochempfindliche Photozellen (Photomultiplier). Deshalb eignen sich diese Geräte besonders gut zur quantitativen Erfassung der bei der Biolumineszenz emittierten Lichtimpulse. TAL und Mitarbeiter (14) haben erstmals 1964 ein solches Zählgerät zur Lumineszenzmessung verwendet. Sie konnten zeigen, daß die Lumineszenz pro Zeiteinheit dem Quadrat der ATPKonzentration proportional ist. Für unsere eigenen Untersuchungen benutzten wir einen FlüssigkeitsSzintillations-Zähler der Fa. Nuclear Chicago. Das Gerät wurde dabei wie für eine Integral-Messung von Tritium eingestellt. Die Messungen wurden wie folgt durchgeführt: 
In einem Probengläschen wurden $0,5 \mathrm{~m} l$ der ATP-haltigen Probe mit einer Tuberkulinspritze $z u \quad 5,0 \mathrm{ml}$ des oben beschriebenen Enzymextraktes gegeben und die Mischung nach leichtem Umschütteln in das Zählgerät eingefahren. Genau 30 Sek. nach $\mathrm{Zu}-$ sammengabe von Probe und Enzymextrakt erfolgte über einen Zeitraum von $0,1 \mathrm{Min}$. die Lumineszenzmessung. Der Meßwert wurde vom Gerät automatisch auf einen Zählstreifen ausgedruckt.

Zur Erfassung der Gesetzmäßigkeiten und möglicher Störfaktoren der Lumineszenzreaktion führten wir folgende Untersuchungen durch:

\section{Die Luminesqenz in Abbängigkeit von der ATP-Konzen- tration}

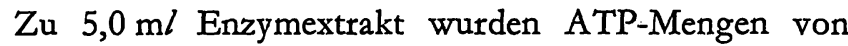
$0,02-10,0 \mu \mathrm{g}$ jeweils in $0,5 \mathrm{ml}$ Glycinpuffer $\mathrm{pH} \mathrm{11,0}$ gelöst gegeben und wie oben beschrieben gemessen. Die ATP-Mengen und die gemessenen Impulswerte (Mittelwerte aus drei Messungen) sowie deren Quadratwurzeln sind in der Tabelle 1 aufgeführt.

Tab. 1

\begin{tabular}{ccc}
\hline $\begin{array}{c}\text { ATP in } \\
\mu \mathrm{g} / 0,5 \mathrm{ml}\end{array}$ & $\begin{array}{c}\text { Lumineszenz in } \\
\text { Imp/0,1 Min. }\end{array}$ & $\sqrt{\text { Impulse/0,1 Min. }}$ \\
\hline 0,02 & 11 & 3,32 \\
0,04 & 42,5 & 6,48 \\
0,06 & 85 & 9,23 \\
0,08 & 160 & 12,65 \\
0,1 & 230 & 15,20 \\
0,2 & 350 & 29,20 \\
0,4 & 8600 & 57,00 \\
0,6 & 15000 & 92,70 \\
0,8 & 22000 & 122,50 \\
1,0 & 70000 & 148,30 \\
2,0 & 262000 & 264,60 \\
4,0 & 492000 & 475,40 \\
6,0 & 665000 & 701,40 \\
8,0 & 730000 & 815,50 \\
10,0 & & 854,40 \\
\hline
\end{tabular}

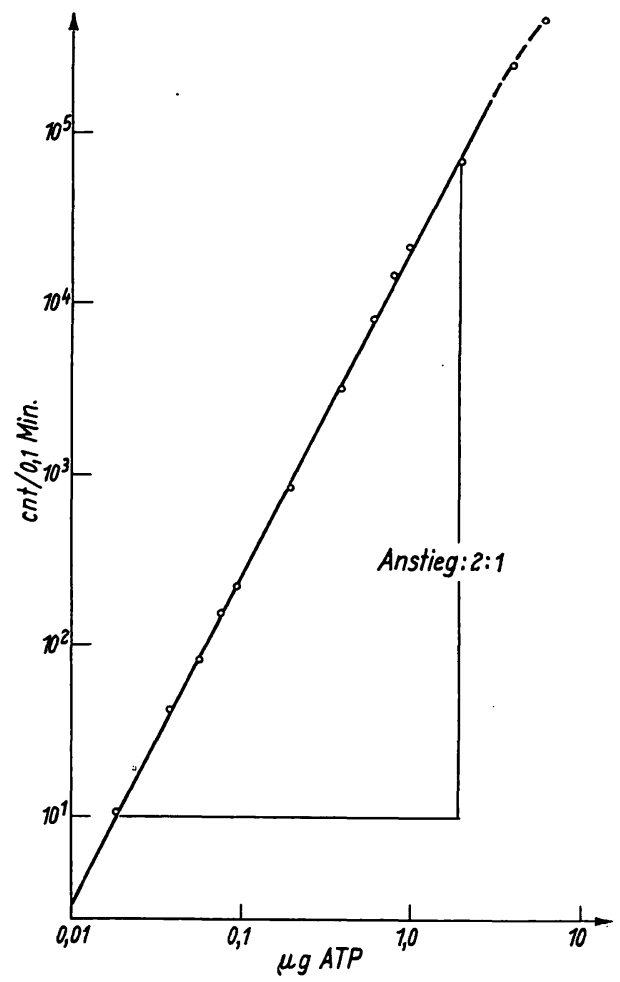

Abb. 3

Darstellung der quadratischen Relation von Lumineszenz und ATP-Konzentration

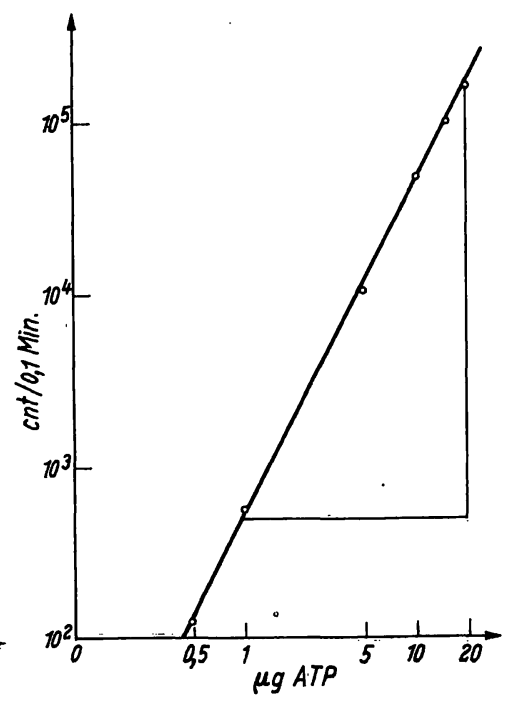

A.bb. 4

Quadratische Abhängigkeit von Lumineszenz und ATP-Konzentration in einem verdünnten Enzymextrakt

Um größere ATP-Konzentrationen (0,5-50 $\mu \mathrm{g}) \mathrm{zu}$ messen) ohne den Meßbereich des Zählgerätes (999999 Impulse) $z u$ überschreiten, verdünnten wir den Enzymextrakt so lange, bis $1 \mu \mathrm{g}$ ATP einen Lumineszenzwert von etwa 500 ergab. Die Meßwerte dieser höheren ATP-Konzentrationen sind in der Tabelle 2 aufgeführt.

Tab. 2

\begin{tabular}{ccc}
\hline $\begin{array}{c}\text { ATP in } \\
\mu \mathrm{g} / 0,5 \mathrm{ml}\end{array}$ & $\begin{array}{c}\text { Lumineszenz in } \\
\text { Impulse/0,1 Min. }\end{array}$ & $\sqrt{\text { Impulse/0,1 Min. }}$ \\
\hline 0,5 & 127 & 11,3 \\
1,0 & 500 & 22,4 \\
5,0 & 11500 & 107,2 \\
10,0 & 52000 & 228,0 \\
15,0 & 108000 & 328,6 \\
20,0 & 170000 & 412,3 \\
30,0 & 390000 & 624,5 \\
50,0 & 750000 & 866,0 \\
\hline
\end{tabular}

Die von uns gemessenen Impulszahlen für die entsprechenden ATP-Konzentrationen sind in den Abbildungen 3 und 4 auf Logarithmenpapier graphisch dargestellt.

Wie aus den Tabellen und Abbildungen ersichtlich, konnten wir die von TAL und Mitarbeitern (14) gefundene quadratische Relation zwischen Impulszahl und ATPKonzentration bestätigen. Die Abweichungen, die sich in beiden Versuchen bei den höchsten Impulszahlen ergạben, sind durch das begrenzte Auflösungsvermögen des Zählgerätes bedingt, das mehrere lichterzeugende Reaktionen, die gleichzeitig oder in einem Zeitintervall unter einer millionstel Sekunde stattfinden, als nur einen Impuls registriert. Aus diesem Grunde wählten wir für unșere weiteren Untersuchungen ATP-Konzentrationen bzw. Enzymextrakte, deren maximale Lumineszenzwerte eine Impulszahl von 250000 pro $0,1 \mathrm{Min}$. nicht überschreiten. Durch entsprechende empirische Verdünnung des Enzymextraktes lassen sich leicht größere Mengen von ATP im günstigsten Meßbereich des Szintillationszählers quantitativ bestimmen.
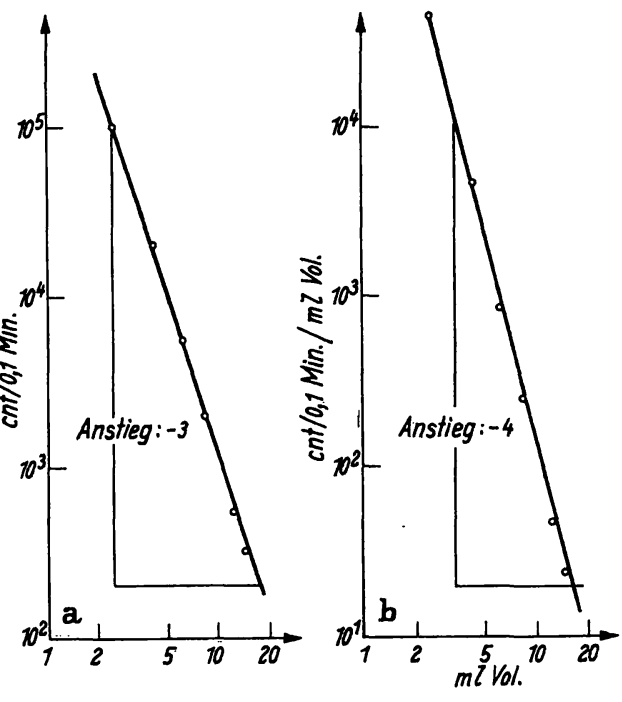

Abb. 5

Abhängigkeit der Lumineszenz von der ATP- und Enzymkonzentration und dem Volumen der Reaktionslösung (Näheres s. Text) 
Die Lumineszenz in Abbängigkeit von der Luciferin-Luciferase-Konzentration

Je 2,0 ml eines Enzymextraktes wurden mit verschiedenen Mengen Arsenatpuffer $\mathrm{pH} 7,4$ verdünnt und diese Mischungen nach Zugabe von $10 \mu \mathrm{g}$ ATP $0,5 \mathrm{~m} /$ Glycinpuffer $\mathrm{pH} 11,0$ in der üblichen Weise gemessen. Dabei konnten wit mit zunehmender Verdünnung und wachsendem Volumen des Reaktionsgemisches eine starke Verminderung der Lumineszenz feststellen. In der folgenden Tabelle sind die Meßwerte aufgeführt.

Tab. 3

\begin{tabular}{|c|c|c|c|c|c|}
\hline $\begin{array}{l}\text { Enzym- } \\
\text { extrakt } \\
\text { in } \mathrm{ml}\end{array}$ & $\begin{array}{c}\text { As- } \\
\text { Puffer } \\
\text { in } \mathrm{ml}\end{array}$ & $10 \underset{\text { in }}{\mu \mathrm{g} l} \underset{\mathrm{mlP}}{\operatorname{Ar}}$ & $\begin{array}{c}\text { Gesamt- } \\
\text { Vol. } \\
\text { in } \mathrm{ml}\end{array}$ & Impulse & $\frac{\text { Impulse }}{\text { Volumen }}$ \\
\hline $\begin{array}{l}2,0 \\
2,0 \\
2,0 \\
2,0 \\
2,0 \\
2,0\end{array}$ & $\begin{array}{r}0 \\
2 \\
4 \\
6 \\
10 \\
12\end{array}$ & $\begin{array}{l}0,5 \\
0,5 \\
0,5 \\
0,5 \\
0,5 \\
0,5\end{array}$ & $\begin{array}{r}2,5 \\
4,5 \\
6,5 \\
8,5 \\
12,5 \\
14,5\end{array}$ & $\begin{array}{r}105000 \\
20500 \\
5800 \\
2100 \\
550 \\
320\end{array}$ & $\begin{array}{r}42000 \\
4550 \\
890 \\
247 \\
46 \\
22\end{array}$ \\
\hline
\end{tabular}

In jedem Meßglas befanden sich also die gleiche Menge ATP $=A$ und die gleiche Menge Enzym = B. Mit zunehmender Verdünnung verminderte sich folglich die Konzentration von $\mathrm{A}$ und $\mathrm{B}$ um den gleichen Faktor $=\frac{1}{\text { Vol. }}$. Unsere Messungen ergaben eine volumenabhängige Abnahme der Lumineszenz (Impulse pro 0,1 Min.) mit der 3. Potenz. Wie wir experimentell feststellen konnten, steigt die Lumineszenz bei konstanter ATP- und Enzymkonzentration mit zunehmendem Volumen linear an. Wurde die im obigen Versuch gemessene Lumineszenz auf ein Volumen von $1,0 \mathrm{~m} l$ bezogen, so ergab sich unter unseren Versuchsbedingungen ein Abfall der Lumineszenz in der 4. Potenz. Unter der bekannten Voraussetzung, daß die Lumineszenz bei konstantem Volumen eine Funktion vom Quadrat der ATP-Konzentration ist, beweist ein Abfall in der 4. Potenz (im obigen Versuch), daß die Lumineszenz gleichzeitig eine Funktion vom Quadrat der Enzymkonzentration ist.

Es gilt also allgemein die Formel:

$$
\begin{aligned}
\frac{\text { Lumineszenz }_{\text {Volumeneinheit }}}{=\mathrm{K} \cdot(\mathrm{ATP}-\text { Konzentration })^{2} \cdot\left(\begin{array}{r}
(\text { Enzymkonzen- } \\
\text { tration })^{2}
\end{array}\right.} \\
=\mathrm{K} \cdot\left(\frac{\mathrm{A}}{\mathrm{V}}\right)^{2} \cdot\left(\frac{\mathrm{B}}{\mathrm{V}}\right)^{2} \\
=\mathrm{K} \cdot \frac{\mathrm{A}^{2} \cdot \mathrm{B}^{2}}{\mathrm{~V}^{4}} \quad \begin{array}{l}
\mathrm{A}=\text { ATP-Menge } \\
\mathrm{B}=\text { Enzym-Menge } \\
\mathrm{V}=\text { Volumen }
\end{array}
\end{aligned}
$$

In der Abbildung 5 sind unsere Untersuchungsergebnisse auf Logarithmenpapier graphisch dargestellt. Figur a zeigt, daß die Lumineszenz bei Verminderung der ATP- und Enzymkonzentration entsprechend der Vergrößerung des Lösungsvolumens mit der 3. Potenz abfällt. Wird die Lumineszenz pro Volumeneinheit berechnet, so erfolgt, wie in Figur b zu sehen, die Abnahme mit der 4. Potenz.

Diese Ergebnisse zeigen deutlich, daß bei der Biolumineszenz exakte Gesetzmäßigkeiten zwischen Konzentration der Reaktionsteilnehmer und Quantität der Lumineszenz bestehen. Gleichzeitig deutet der Nach- weis dieser Gesetzmäßigkeit darauf hin, daß bei unseren Versuchen keinerlei Störungen durch andere mögliche Faktoren auftraten.

\section{Untersuchungen zur ATP-Spezifität der Luninineszenzreaktion}

Um zu prüfen, ob das von uns verwendete LuciferinLuciferase-System nur mit ATP oder auch mit anderen energiereichen organischen Phosphaten reagiert, untersuchten wir die Verbindungen: Uridintriphosphat (UTP), Adenosindiphosphat (ADP) und Creatinphosphat (CP). Die Substanzen wurden einzeln oder kombiniert in verschiedenen Konzentrationen in Glycinpuffer gelöst und mit dem Enzymextrakt in beschriebener Weise gemessen. Die Untersuchungen ergaben, daß UTP und CP nicht mit dem Enzymsystem reagierten. Beim ADP konnten wir dagegen eine Lumineszenzreaktion nachweisen, die allerdings $30 \mathrm{mal}$ schwächer war als für die gleiche Menge ATP. Eine Mischung von $\mathrm{ADP}$ und $\mathrm{CP}$ erhöhte den für $\mathrm{ADP}$ allein gefundenen Wert nicht, so daß man die mögliche Reaktion:

$\mathrm{ADP}+$ Creatinphosphat $\rightleftarrows \mathrm{ATP}+$ Creatin

ausschließen konnte. Äquimolare Mengen von ATP und ADP führten zu Meßwerten, die um nur 3,3\% über den entsprechenden ATP-Werten lagen. Ob das Enzymsystem mit ADP reagiert, oder ob die geringe Lumineszenz des ADP durch Spuren von ATP als Verunreinigung verursacht wurde, haben wir nicht abgeklärt. Außerdem ist es nicht ausgeschlossen, daß der Enzymextrakt aus den Glühwürmchenschwänzen eine ADP-Phosphomutase enthält, die die Reaktion:

$$
2 \mathrm{ADP} \rightleftarrows \mathrm{AMP}+\mathrm{ATP}
$$

ermöglicht. $\mathrm{Da}$ die Lumineszenz, hervorgerufen durch ATP, jedoch $30 \mathrm{mal}$ stärker ist, dürften sich mit diesem Meßsystem Veränderungen des ADP-Gehaltes in biologischem Material gegenüber solchen von ATP kaum bemerkbar machen.

\section{Untersuchung möglicher Störfaktoren}

Wie bei den meisten enzymatischen Reaktionen können auch beim Luciferin-Luciferase-System bestimmte Störfaktoren auftreten. Eingehende Untersuchungen $z u$ diesem Thema sind vor allem von MCELROY und STREHLER (10) angestellt worden. Dabei wurden die Einflüsse von Schwermetallen, $\mathrm{pH}$, Temperatur, Ionenkonzentration und besonderen Inhibitoren wie 2-Methyl-1,4-naphthochinon, Natriumazid und Urethan studiert. In unseren eigenen Experimenten fanden wir für die Lumineszenzreaktion einen $0,1 \mathrm{M}$ Arsenatpuffer $\mathrm{pH} 7,4$ und eine $\mathrm{Mg}^{++}$-Konzentration von $0,02 \mathrm{M}$ als optimal. Der $\mathrm{pH}$-Wert des Arsenatpuffers wurde auch durch Zugabe von $0,5 \mathrm{~m} l$ Glycinpuffer $\mathrm{pH} 11,0$ (Probenlösung) zu $5,0 \mathrm{~m} l$ Puffer nicht verändert. Temperaturschwankungen im Rahmen der Zimmertemperatur zwischen 20 und $25^{\circ}$ konnten vernachlässigt werden, zumal bei allen Messungen stets mit einem ATP-Standard gleicher Temperatur verglichen wurde. Größere Mengen von $\mathrm{Mg}^{++}, \mathrm{Ca}^{++}$und $\mathrm{Cl}^{-}$-Ionen verminderten die Lumineszenz. $\mathrm{Da}$ der von uns untersuchte Pfotenextrakt vor der Messung noch 1: 5 mit Glycinpuffer verdünnt wurde, traten hierbei keinerlei Störungen durch mitextrahierte Substanzen aus dem Pfotengewebe auf. Bei Verabreichung von Pharmaka oder anderen Wirkstoffen wurde der Einfluß dieser Substanzen in den jeweiligen Konzentrationen geprüft. Für die Untersuchung anderer Gewebe oder Materialien ist es zu empfehlen, mit der , internal standard“Methode durch Zusatz einer bestimmten ATP-Menge zum Ge- 
webeextrakt den Einfluß möglicher Störfaktoren quantitativ zu ermitteln. Außerdem ist in manchen Fällen ein chemischer oder Farb-Quench möglich, der zu einer verminderten Impulsausbeute führt und dessen Ausmaß durch Aufstellung einer Quench-Kurve berechnet werden kann, wie dies bei der Messung von radioaktiven Substanzen mit dem Flüssigkeits-Szintillationszähler üblich ist.

\section{Diskussion}

Die beschriebene Extraktions- und Bestimmungsmethode von ATP in biologischem Material zeichnet sich vor allem durch eine hohe Empfindlichkeit und gute Reproduzierbarkeit aus. Die ATP-Extrakte können ohne Verlust durch Hydrolyse lange bei Zimmertemperatur aufbewahrt werden. Bei gleichbleibender Empfindlichkeit lassen sich mit der Lumineszenzmethode weite Konzentrationsbereiche von ATP erfassen. Während bei Untersuchungen über Veränderungen des ATP-
Gehaltes im Gewebe mitextrahierte ADP-Mengen die quantitativen Vergleiche kaum beeinflussen, muß für die Absolut-Bestimmungen von ATP das vorhandene ADP mit einer anderen Methode bestimmt und entsprechend subtrahiert werden. Diese ADP-Bestimmung kann z. B. mit dem Biochemica Test Boehringer durchgeführt werden. Durch die Verwendung eines Flüssigkeits-Szintillationszählers anstelle der bisher üblichen Photometer zur Messung der Lumineszenz können der Verbrauch von Glühwürmchenschwänzen auf ein Minimum reduziert und damit die Kosten der ATP-Bestimmung wesentlich verringert werden. Inzwischen wurden von uns mit Hilfe der beschriebenen Methode ATPMessungen im Blut, Gehirn, Mastzellen und Knorpel mit Erfolg durchgeführt, was die Zuverlässigkeit des Verfahrens bei verschiedenstem biologischem Material bestätigt.

\title{
Literatur
}

1. Bergmeyer, H. U., Methoden der enzymatischen Analyse. Verlag Chemie GmbH, Weinheim/Bergstraße (1962). - 2. HurLbert, R. B., H. Schmitz, A. E. Brumm und V. R. Potrer, J. biol. Chemistry 209, 23 (1954). - 3. Cohn, W. E. und C. E. Carter, J. Amer. chem. Soc. 72, 4273 (1950). - 4. Kalbhen, D. A., Arch. internat. pharmacodyn. thérap. 143, 362 (1963). - 5. Wirthaus, G., Med. Pharmocol. exp. 15, 432 (1966). - 6. Berger, L., M.W. Slein, S. P. Colowick und C. E. Cori in: J. Gen. Physiol. 29, 379 (1946). - 7. Strehler, B. L. und J. R. Totter, Arch. Biochem. 40, 28 (1952). - 8. Dubois, R., zit. bei A. M. Chase, Methods biochem. Analysis, 8, 61 (1960). - 9. McErroy, W. D., Proc. Natl.
Acad. Sci. U. S. 33, 342 (1947), zit. bei A. M. Chase, Methods biochem. Analysis, 8, 61 (1960). - 10. McElroy, W. D. und B. L. Strehler, Arch. Biochem. 22, 420 (1949). - 11. Green, A. A. und W. D. McElroy, Crystalline Firefly Luciferase. Biochim. biophysica Acta (Amsterdam) 20, 170 (1956). - 12. Brtrer, B. und W. D. McElroy, Arch. Biochem. Biophysics 72, 358 (1957). 13. Strehler, B. L. und W. D. McElroy, Assay of adenosine triphosphate. In: Methods in Enzymology, Bd. III, S. 871, Academic Press - New York (1957). - 14. TAL, E., S. Dikstein und F. G. Sulman, Experientia, Basel 20, 652 (1964). -

\section{Eine Methode der automatischen Bestimmung des Gesamt-Eisen in Blutserum und Urin mit 2,6-Di-[pyridyl-(2)]-4-[p-methoxy-phenyl]-pyridin und seiner Sulfosäure}

\author{
Von R. Schmidt, W. Weis, V. KLingmülleR und HJ. StaudingeR ${ }^{1}$ )
}

Aus dem Pbysiologisch-Chemischen Institut der Justus-Liebig-Universität, Gießen (Direktor: Prof. Dr. Hj. Staudinger). und dem Klinisch-Chemischen Institut, Mannbeim (Direktor: Prof. Dr. Dr. V. Klingmüller)

(Eingegangen am 20. März 1967)

Es wird eine direkte Bestimmung des Serumeisens im Autoanalysator mit der Sulfosäure des neusynthetisierten 2,6-Di-[pyridyl-(2)]-4[p-methoxy-phenyl]-pyridin in allen Einzelheiten beschrieben. Dieser Komplexbildner besitzt einen sehr hohen molaren Extinktionskoeffizienten von $\varepsilon=26900$. Die Methode entspricht den Bedürfnissen der klinischen Diagnostik und erlaubt es, in $60 \mathrm{Min} .60 \mathrm{Bestimmun}$ gen durchzuführen. Serumproteine, Serumfarbstoffe usw. stören nicht. Das Reagenz bleibt übẹ Monate konstant. Richtigkeit, Reproduzierbarkeit, Spezifität und Empfindlichkeit werden kritisch untersucht.

Full practical details are given for a direct determination of iron in serum by an autoanalyzer technique, which employs the sulfonic acid derivate of the newly synthesized 2,6-Di-[pyridyl-(2)]-4-[p-methoxy-phenyl]-pyridine. The new complexing agent possesses a high molar extinction coefficient of $\varepsilon=26,900$. The complete procedure corresponds to the needs of clinical diagnosis and requires 60 min. for 60 determinations. Serum proteins, dye-stuffs etc. do not interfere. The reagent is stable for months. Accuracy, reproducibility, specifity and sensitivity were examined critically.

Die Forderungen der modernen klinisch-chemischen Differential-Diagnostik nạch einer zuverlässigen Eisenbestimmung $(1,2)$ führten dazu, daß bereits eine Reihe von Modifikationen im Autoanalyzer ("Technicon")

1) Auszugsweise vorgetragen auf dem 6. Internat. Kongr. Klin. Chem., München, 26.-30. Juli 1966. vorgeschlagen wurden: DeLcour 1964 (3); FüHR 1964 u. 1964 (4); HeNRIKSEN 1965 (5); LANDERS 1958 (6); LePpla und Mitarbeiter 1963 (7); Summers und Mitarbeiter 1964 (8); STEINER 1965 (9); ZAK und Mitarbeiter 1964 (10). Als KRÖHNKE, STAUDINGER und Mitarbeiter (11) das neue Tripyridyl-Derivat 2,6-Di- 